\title{
Conservative Management of Presumed Fetal Anemia Secondary to Maternal Chemotherapy for Acute Myeloid Leukemia
}

\author{
Christina M. Nowik, MD, MPA, FRCSC ${ }^{1}$ Alina S. Gerrie, MD, MPH, FRCPC 2 \\ Jonathan Wong, MD, MSCCH, FRCPC 3
}

${ }^{1}$ Department of Obstetrics and Gynaecology, University of British Columbia, Vancouver, British Columbia, Canada

2 Division of Hematology and Medical Oncology, University of British Columbia, BC Cancer Agency, Vancouver, British Columbia, Canada

${ }^{3}$ Division of Neonatology, University of British Columbia, Vancouver, British Columbia, Canada

\begin{abstract}
Address for correspondence Christina Nowik, MD, MPA, FRCSC, C400-4500 Oak Street, Vancouver, BC V6H 3N1, Canada (e-mail: christina.nowik@cw.bc.ca).
\end{abstract}

AJP Rep 2021;11:e137-e141.

\begin{abstract}
Acute myeloid leukemia occurs rarely during pregnancy. When it is diagnosed remote from term, treatment in the form of daunorubicin plus cytarabine induction with consolidative cytarabine is typically undertaken after the first trimester. There is little data to guide fetal monitoring, in particular, whether and how often middle cerebral artery peak systolic velocity (MCA PSV) should be measured to screen for fetal anemia. Cytarabine may be particularly myelosuppressive to the fetus, but information pertaining to the management of this complication is also lacking in published

Keywords

- leukemia

- myeloid

- acute pregnancy

- ultrasonography

- Doppler fetal monitoring literature. To our knowledge, we present the first case of presumed severe fetal anemia related to in utero exposure to chemotherapy that was managed conservatively with close sonographic monitoring, including serial measurement of MCA PSV. This case suggests that in the absence of hydrops fetalis or other signs of fetal decompensation, expectant management with ultrasound twice weekly, including MCA PSV, is appropriate. Ultrasounds may be decreased to weekly when MCA PSV does not suggest fetal anemia. Screening for fetal anemia can provide helpful information to guide the timing of chemotherapy administration and delivery.
\end{abstract}

\section{Key Points}

- Chemotherapy for acute myeloid leukemia can cause fetal anemia.

- Fetal MCA PSV can be used to safely and effectively screen for fetal anemia.

- Observation of fetal anemia due to chemotherapy is reasonable, in the absence of hydrops.

- Monitoring of fetal MCA PSV can help guide timing of chemotherapy and delivery.

received

July 9,2020

accepted after revision

October 8, 2021
DOI https://doi.org/

$10.1055 / \mathrm{s}-0041-1740561$. ISSN 2157-6998. (c) 2021. The Author(s).

This is an open access article published by Thieme under the terms of the Creative Commons Attribution-NonDerivative-NonCommercial-License, permitting copying and reproduction so long as the original work is given appropriate credit. Contents may not be used for commercial purposes, or adapted, remixed, transformed or built upon. (https://creativecommons.org/ licenses/by-nc-nd/4.0/)

Thieme Medical Publishers, Inc., 333 Seventh Avenue, 18th Floor, New York, NY 10001, USA 
Leukemia affects approximately one in 75,000 to one in 100,000 pregnancies, the majority of which are acute leukemia. Approximately two-thirds of these are acute myeloid leukemia (AML). ${ }^{1}$ Standard induction chemotherapy generally consists of daunorubicin plus cytarabine on a $7+3$ schedule with high-dose cytarabine and/or hematopoietic stem cell transplant (HSCT) consolidative therapy..$^{1-3}$ Although cytotoxic therapy in the second and third trimesters has been associated with intrauterine growth restriction, intrauterine fetal demise, and neonatal myelosuppression, treatment delays may pose unacceptable risks to the mother. ${ }^{1,2}$ Therefore, balancing the anticipated impacts of in utero exposure to cytotoxic drugs with risks of prematurity, chemotherapy during pregnancy is generally undertaken if AML is diagnosed remote from term. ${ }^{1,4}$

Given the rarity of the diagnosis and treatment of AML during pregnancy, standards for fetal monitoring are not well established. The British Society for Haematology recommends ultrasounds every 2 weeks to assess fetal growth and well-being. ${ }^{1}$

However, in utero exposure to cytarabine may pose an additional risk of fetal and neonatal anemia. While anthracyclines, such as daunorubicin, are thought to have limited placental transfer (5-7\% in primate studies), ${ }^{5}$ cytarabine has wide tissue distribution and may have significantly higher placental transfer (22.6\% in mouse studies). ${ }^{6}$ Cytarabine is an antimetabolite and is associated with prolonged myelosuppression. ${ }^{7}$ Parrott and Holland described two cases of neonatal anemia suspected antenatally based on abnormal or borderline middle cerebral artery peak systolic velocity (MCA PSV) after in utero exposure to cytarabine. $^{2}$

Measurement of the fetal MCA PSV is a highly sensitive test for moderate-to-severe fetal anemia. ${ }^{8}$ MCA PSV greater than 1.5 multiples of the median (MoM) has been reported to have up to $100 \%$ sensitivity for moderate-to-severe anemia, with a false positive rate of $12 \%{ }^{8}$ In a review article in the European Journal of Haematology, Thomas recommends screening for fetal anemia before and after chemotherapy. ${ }^{9}$ In the case series by Parrott and Holland, MCA PSV was measured weekly. ${ }^{2}$

It is not clear how suspected fetal anemia should be managed. In the aforementioned case series by Parrott and Holland, both cases were delivered emergently for abnormal fetal heart rate shortly after MCA PSV became abnormal. ${ }^{2}$ We found only one case report in the published literature of intrauterine transfusion (IUT) performed in the context of chemotherapy-induced fetal anemia. In that case, the authors described finding sonographic evidence of fetal ascites and anemia. ${ }^{10}$ The potential benefits of treating fetal anemia with IUT must be weighed against procedure-related fetal loss, where rates have been reported in the range of 0.9 to $4.9 \%$ per IUT. $^{11}$

As neonates exposed to maternal chemotherapy may be pancytopenic, it could be anticipated that a fetus with chemotherapy-induced anemia might be thrombocytopenic. Neonates who have been exposed to in utero chemotherapy are at highest risk of myelosuppression in the first 28 days after administration of chemotherapy. ${ }^{12}$ Udink Ten Cate et al described a case in which a 33-week baby was delivered 1 week after chemotherapy due to premature prelabor rupture of membranes and maternal bacteremia. The neonate required platelet transfusion. ${ }^{13}$ Thrombocytopenia could increase the risk of complications from IUT, specifically significant bleeding from the venipuncture site.

Furthermore, the fetus would be expected to undergo bone marrow recovery as the mother does, and therefore, the anemia would also be expected resolve. However, depending on gestational age and comorbidities (i.e., prematurity), the degree of anemia and time to recovery would be variable and could have significant clinical implications. To our knowledge, we present the first published case of conservative management of presumed chemotherapy-induced severe fetal anemia.

\section{Case Report}

A 23-year-old G1P0 was diagnosed incidentally with AML on routine prenatal bloodwork. Routine complete blood count (CBC) at 14 weeks and 0 days gestation (based on first trimester ultrasound dating) revealed hemoglobin $113 \mathrm{~g} / \mathrm{L}$, mean corpuscular volume $99.1 \mathrm{fL}$, white blood cells $16.2 \times 10^{9} / \mathrm{L}$, neutrophils $13.0 \times 10^{9} / \mathrm{L}$, monocytes $0.13 \times 10^{9} / \mathrm{L}$, circulating blasts $1.95 \times 10^{9} / \mathrm{L}$ with Auer rods present, and platelets $119 \times 10^{9} / \mathrm{L}$. The patient had only noted recent mild nausea and fatigue, thought to be consistent with early pregnancy. Her past medical history was remarkable for mild asthma and a history of pyelonephritis 1 year prior to presentation. Her only medications or supplements were prenatal vitamin and an iron supplement.

Bone marrow aspirate and biopsy revealed AML, with karyotype showing somatic monosomy $\mathrm{X}$ and between 2 and 23 double minute chromosomes. Fluorescence in situ hybridization showed MYC amplification of $83 \%$, likely originating from the double minute chromosomes. Myeloid gene panel demonstrated an EZH2 mutation with a variant allele frequency of $86 \%$.

The patient was admitted to an inpatient leukemia unit where she received standard $7+3$ cytarabine $100 \mathrm{mg} / \mathrm{m}^{2}$ and daunorubicin $60 \mathrm{mg} / \mathrm{m}^{2}$ starting at 14 weeks and 6 days. During her admission, she developed a left lower molar dental infection with pericoronitis and culture-negative febrile neutropenia. This was treated with meropenem, cefuroxime, and metronidazole until tooth extraction was completed. Amphotericin B liposomal formulation was used for fungal prophylaxis, given the known teratogenic risks associated with azoles.

Subsequent bone marrow biopsy demonstrated less than $5 \%$ blasts, consistent with a complete remission. Given the high-risk cytogenetic and molecular features, consolidative HSCT was planned, although the decision was made to delay HSCT until after delivery, if feasible. Bridging consolidative therapy with intermediate-dose cytarabine $\left(1000 \mathrm{mg} / \mathrm{m}^{2}\right.$ intravenously daily $\times 5$ days) was therefore initiated at 20 weeks gestation.

Regular assessment of MCA PSV was started at 18 weeks gestation. Screening for fetal anemia is not typically done 
prior to 18 weeks because IUT generally cannot be technically completed at earlier gestations. At 18 weeks and 6 days, the MCA PSV was $1.91 \mathrm{MoM}$ ( $>1.5 \mathrm{MoM}$ is suggestive of moderate-severe anemia). The fetus was otherwise normally grown with normal anatomic assessment. The amniotic fluid volume was normal and there were no signs of hydrops fetalis. We decided to proceed with close monitoring, with a plan to reconsider IUT if the fetus started to show signs of decompensation (e.g., hydrops fetalis, cardiomegaly).

We repeated ultrasounds, including MCA PSV, twice per week while the MCA PSV was greater than 1.5 MoM. The fetus never became hydropic. We specifically monitored for the evidence of intracranial hemorrhage, which was never noted. Once the MCA PSV normalized, we reverted to once weekly ultrasound. The patient had fetal nonstress tests daily while admitted to hospital and twice per week while an outpatient.

Subsequent cycles of chemotherapy were delayed, within the limits of what was felt to be clinically acceptable, to allow for fetal bone marrow recovery. The patient received a total of two cycles of intermediate dose cytarabine at 20 weeks and 3 days and 26 weeks and 0 days.

As shown in - Fig. 1, MCA PSV increased predictably following each administration of chemotherapy, but normalized within 5 weeks. It should be noted that normalization of fetal MCA PSV was preceded by normalization of maternal white blood cell count after each cycle of chemotherapy.

Delivery was organized for approximately 32 weeks gestation, allowing 6 weeks for maternal and fetal bone marrow to recover after the last cycle of chemotherapy prior to delivery. We decided to deliver prematurely rather than pursue another cycle of consolidation chemotherapy, to expedite definitive maternal treatment with HSCT.

The patient received two doses of intramuscular betamethasone for fetal lung maturity at 31 weeks gestation. She was admitted to hospital at 31 weeks and 4 days and received cervical ripening in the form of an intravaginal dinoprostone insert over 48 hours, transcervical foley catheter over 24 hours, and two doses of intravaginal dinoprostone gel before commencing oxytocin induction of labor with intravenous magnesium for fetal neuroprotection. She had a spontaneous vaginal delivery of a female infant weighing $1,770 \mathrm{~g}$ (50th percentile) at 32 weeks and 1 day gestational age. One minute of delayed cord clamping was performed.

The infant's Apgar scores were 5 at 1 minute, 8 at 5 minutes, and 9 at 10 minutes. She required 2 minutes of intermittent positive pressure ventilation and was then transitioned to continuous positive pressure ventilation. Initial CBC revealed a slightly low hemoglobin at $131 \mathrm{~g} / \mathrm{L}$, white blood cells $14.7 \times 10^{9} / \mathrm{L}$ with neutrophils $7.109 / \mathrm{L}$, and

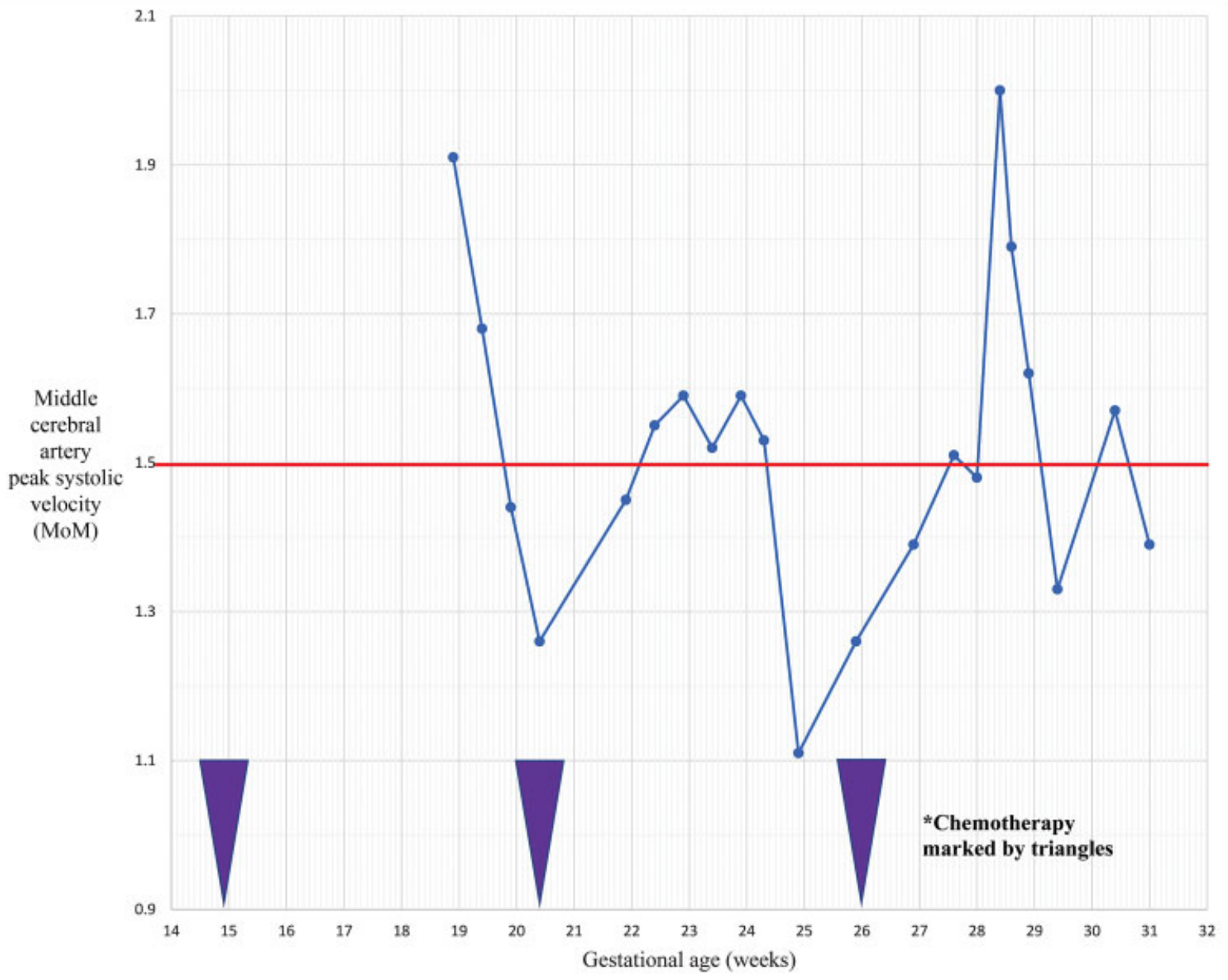

Fig. 1 Fetal middle cerebral artery peak systolic velocity in relation to gestational age and chemotherapy. 
platelets $306 \times 10^{9} / \mathrm{L}$. Blood cultures were negative. No transfusions were required during her admission to the neonatal intensive care unit. Neonatal echocardiogram was performed due to theoretical potential for cardiomyopathy related to daunorubicin exposure. Echocardiogram showed normal structure and function; a repeat scan is planned in 1 year.

The infant remained neurologically appropriate through her admission. Head ultrasound performed on day of life 10 revealed nonspecific periventricular white matter changes. Magnetic resonance imaging of the head was performed at term, revealing periventricular white matter $\mathrm{T} 1$ hyperintense foci without restricted diffusion, consistent with nonspecific sequelae of white matter injury. Clinical neurological examination at 1 month corrected age was within normal limits and follow-up continues.

The patient went on to receive a myeloablative allogenic HSCT from a 10/10 human leukocyte antigen allele-matched unrelated male donor. Conditioning chemotherapy was with intravenous busulfan and cyclophosphamide. The donor was cytomegalovirus (CMV) nonreactive and the recipient was CMV reactive. Her posttransplant course was complicated by CMV reactivation, treated with valganciclovir, and grade 1 acute graft-versus-host disease of the skin, treated with prednisone and basiliximab. Bone marrow aspirate and biopsy at 100 days after transplant confirmed ongoing complete remission. At 6 months postpartum, the patient was otherwise clinically well without evidence of recurrence.

\section{Comment}

In utero exposure to chemotherapy for AML has been associated with fetal anemia, and recent publications advocate for regular screening with MCA PSV. ${ }^{1,2,4,9}$ We have presented a case of presumed severe fetal anemia, diagnosed on the basis of persistent and significant elevations of the MCA PSV above $1.5 \mathrm{MoM}$, which was managed conservatively. Operating under the assumption that fetal myelosuppression would be a self-limited reaction to chemotherapy, and that procedure-related risks would be increased due to fetal thrombocytopenia, we endeavored to closely monitor for signs of fetal decompensation, rather than to offer IUT for severe fetal anemia. After each cycle of chemotherapy, the MCA PSV normalized, and we never observed evidence of hydrops fetalis or intracranial hemorrhage. The infant was delivered after the last normalization of MCA PSV; she required no transfusions and had no infectious complications during her admission.

Evidence of periventricular white matter injury was observed in the infant. It is reassuring that the infant has been neurologically appropriate. While it cannot be ruled out that this finding is related to in utero chemotherapy, it is a known complication of preterm birth.

Other remaining questions include the ideal frequency of fetal monitoring, including ultrasounds and nonstress tests, and the value of screening for anthracycline-induced cardiomyopathy.
While further evidence is required to provide strong guidance about the management of fetal anemia in the context of maternal AML treatment, this case report suggests that conservative management with close sonographic observation is reasonable, as long as there are no signs of hydrops fetalis or fetal decompensation. If such signs had presented, we would have pursued IUT or delivery, depending on gestational age.

Monitoring of MCA PSV can be used to time subsequent cycles of chemotherapy. Close collaboration between maternal-fetal medicine and hematology was crucial to coordinate modifications to the patient's chemotherapy schedule. Cycles of consolidative cytarabine were given approximately 6 weeks after the previous cycle. This allowed for the severe fetal anemia to resolve, and was felt to be a reasonable compromise that would not unduly affect maternal treatment.

Furthermore, while the incidence of neonatal cytopenias is low when delivery occurs more than 29 days after chemotherapy in general, ${ }^{12}$ there are theoretical concerns that cytarabine may be particularly associated with increased incidence and duration of fetal myelosuppression. ${ }^{2}$ In our case, MCA PSV was still elevated above $1.5 \mathrm{MoM} 4$ weeks after chemotherapy, and remained elevated beyond normalization of maternal cell counts. Regular monitoring of MCA PSV allowed us to ensure fetal bone marrow recovery prior to delivery.

\section{Conflict of Interest}

Dr. Gerrie reports personal fees and other from Janssen, personal fees and other from AbbVie, other from Roche, personal fees and other from Astrazeneca, personal fees from Sandoz, personal fees from Celgene, outside the submitted work.

\section{References}

1 Ali S, Jones GL, Culligan DJ, et al; British Committee for Standards in Haematology. Guidelines for the diagnosis and management of acute myeloid leukaemia in pregnancy. Br J Haematol 2015;170 (04):487-495

2 Parrott J, Holland M. Undetected severe fetal myelosuppression following administration of high-dose cytarabine for acute myeloid leukemia: is more frequent surveillance necessary? Case Rep Obstet Gynecol 2017;2017:5175629

3 Horowitz NA, Henig I, Henig O, Benyamini N, Vidal L, Avivi I. Acute myeloid leukemia during pregnancy: a systematic review and meta-analysis. Leuk Lymphoma 2018;59(03):610-616

4 Fracchiolla NS, Sciumè M, Dambrosi F, et al. Acute myeloid leukemia and pregnancy: clinical experience from a single center and a review of the literature. BMC Cancer 2017;17(01):442

5 Esposito S, Tenconi R, Preti V, Groppali E, Principi N. Chemotherapy against cancer during pregnancy: a systematic review on neonatal outcomes. Medicine (Baltimore) 2016;95(38):e4899

6 Van Calsteren K. Chemotherapy during pregnancy: pharmacokinetics and impact on foetal neurological development. Facts Views Vis ObGyn 2010;2(04):278-286

7 Stentoft J. The toxicity of cytarabine. Drug Saf 1990;5(01):7-27

8 Mari G, Norton ME, Stone J, et al; Society for Maternal-Fetal Medicine (SMFM). Electronic address: pubs@smfm.org. Society for Maternal-Fetal Medicine (SMFM) Clinical Guideline \#8: the 
fetus at risk for anemia-diagnosis and management. Am J Obstet Gynecol 2015;212(06):697-710

9 Thomas X. Acute myeloid leukemia in the pregnant patient. Eur J Haematol 2015

10 Hoopmann M, Rahimi G, Hartlapp I, Eifinger F, Garnier Y, Bald R. [Chemotherapy-induced fetal anemia in maternal acute myelocytic leukemia]. Ultraschall Med 2008;29(04):424-427

11 Lindenburg IT, van Kamp IL, Oepkes D. Intrauterine blood transfusion: current indications and associated risks. Fetal Diagn Ther 2014;36(04):263-271
12 La Nasa M, Gaughan J, Cardonick E. Incidence of neonatal neutropenia and leukopenia after in utero exposure to chemotherapy for maternal cancer. Am J Clin Oncol 2019;42(04): 351-354

13 Udink ten Cate FE, ten Hove CH, Nix WM, de Vries JI, van de Loosdrecht AA, van Elburg RM. Transient neonatal myelosuppression after fetal exposure to maternal chemotherapy. Case report and review of the literature. Neonatology 2009;95(01): $80-85$ 